\title{
Zur Entstehungsgeschichte der österreichischen Grundrechte Vom Ur-Entwurf Eduard Sturms zum Staatsgrundgesetz über die allgemeinen Rechte der Staatsbürger 1867
}

\begin{abstract}
The reconstruction of the Hungarian Constitution of 1848 - following the "Compromise" of 1867 between the monarch and the Hungarian Diet - induced the Cisleithanian parliament likewise to return to former constitutional concepts. A Constituant Committee, set up by the House of Representatives of the Austrian Imperial Council, was guided along the content of the Imperial Constitution, brought into force with oktroy in 1849. The liberal German representative Eduard Sturm, attorney from Brno in Moravia, was appointed to prepare a draft about fundamental rights. A subcommittee of the Constituant Committee brought Sturm's draft to perfection. In essence only those fundamental rights enacted by an oktroy just in 1849 were left behind. Sturm pithily jotted down his notes about the results of the discussion within the Constituant Committee: "Everything 1849"!
\end{abstract}

\section{Allgemeines ${ }^{1}$}

Das Staatsgrundgesetz über die allgemeinen Rechte der Staatsbürger war im Dezember 1867 als eines von insgesamt sieben Verfassungsgesetzen ins Leben getreten. Es blieb bis zum Ende der Monarchie ein unveränderter Bestandteil der Verfassungsordnung. Nach dem Zerfall der Monarchie wurde es gemäß $\S 16$ Staatsgründungbeschluss vom 30. Oktober 1918 ein impliziter Bestandteil der provisorischen Verfassungsordnung von Deutschösterreich. Es beeinflusste die 1919/20 laufenden Arbeiten an der

\footnotetext{
*Anmerkung des Verfassers: In Vorbereitung begriffen ist die Veröffentlichung einer Edition mit allen für die Textgenese des Staatsgrundgesetzes maßgeblichen Entwürfen und anderen sich darauf beziehenden zeitgenössischen Materialien; für diese Edition wird der vorliegende Beitrag die Grundlage einer umfassenden Einleitung bilden.

${ }^{1}$ Zum Folgenden BRAUNEDER, Historische Entwicklung der modernen Grundrechte.
}

definitiven Verfassung, insbesondere auch die Vorentwürfe von Hans Kelsen, auf deren Grundlage 1920 das B-VG für die Republik Österreich ausgearbeitet wurde. Aufgrund der weltanschaulichen Gegensätze der politischen Parteien kam in der Konstituierenden Nationalversammlung jedoch keine Einigung über einen neuen Katalog von Grundrechten zustande. Es war daher notwendig, die Grundrechte des Staatsgrundgesetzes von 1867 beizubehalten: Sie wurden gemäß Art. 151 B-VG zum Bestandteil des Bundes-Verfassungsgesetzes erklärt.

Die Wurzeln der Grundrechte des Staatsgrundgesetzes von 1867 liegen ihrerseits in den frühkonstitutionellen Verfassungen des Kaisertums Österreich von 1848 und 1849 sowie im Entwurf des Konstituierenden Reichstags von Kremsier 1848/49; mit der Beendigung des Frühkonstitutionalismus wurden mit Jahresbeginn 1852 auch die Grundrechte weitgehend aufgehoben. Nach dem Neoabsolutismus und der Periode einer ständisch beschränkten Monarchie kam es 1867 
zur Wiederherstellung des Konstitutionalismus und damit auch zur der Restauration der Grundrechte. Den entscheidenden Anstoß dazu gab der "Ausgleich" über die verfassungsrechtliche Stellung der ungarischen Provinzen, wodurch sich die Österreichische Monarchie in zwei Verbände von Ländern mit unterschiedlicher Verfassungsordnung spaltete. ${ }^{2}$

\section{Verfassungsrechtliche Bedingungen in Cisleithanien nach dem Ausgleich mit Ungarn}

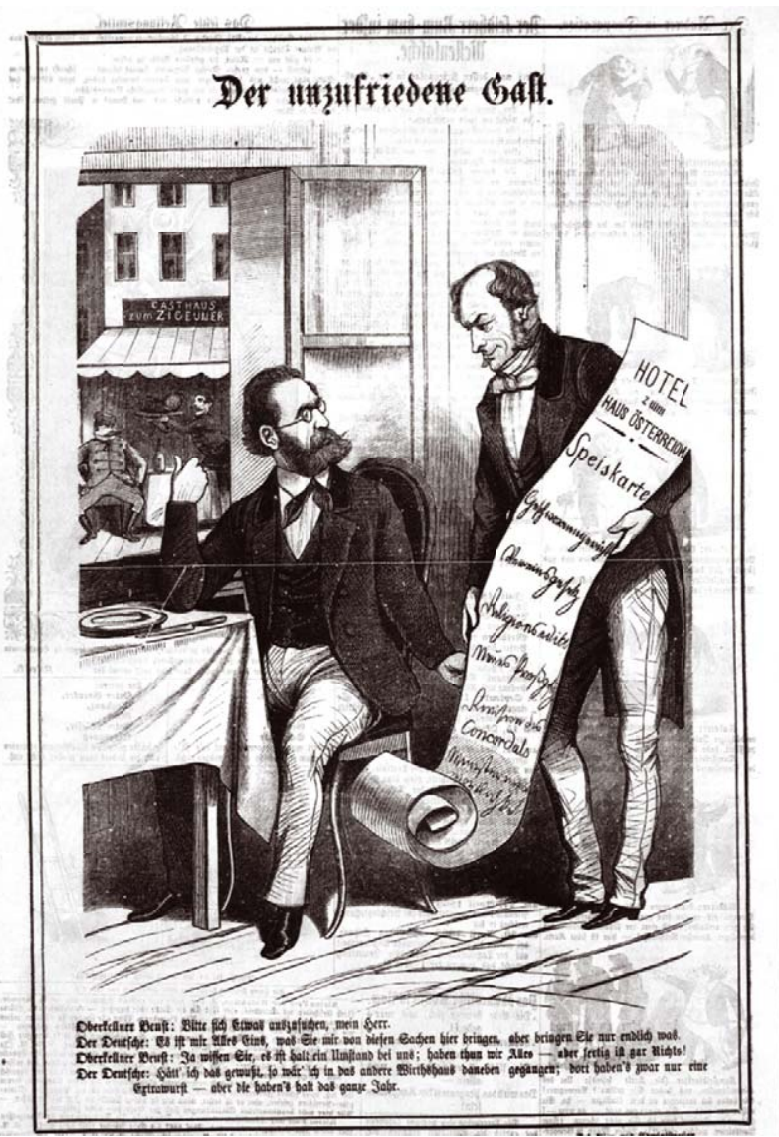

Am 11. Juli 1867 erschien in Wien im „Kikeriki“, diese Karikatur: Sie zeigt Friedrich Beust, den für den Ausgleich mit Ungarn und für die Regierungsvorlagen zur Verfassungsrevision in Cisleithanien verantwortlichen Ministerpräsi-

${ }^{2}$ AÖRG, Rechts- und Verfassungsgeschichte, 202-221; zu den Grundrechten 1848/49: ebd. 203, 208, $210 f$. denten, als Oberkellner im „Hotel zum Haus Österreich“. Er reicht einem Gast, „Der Deutsche“ genannt, als Speisekarte einen Katalog mit konstitutionellen Forderungen an die Verfassungsreform: "Geschwornengerichte" - „Vereinsgesetz" - "Religionsedikte“ - "Neues Preßgesetz" - "Revision des Concordats" - „Ministerverantwortlichkeit" etc.

Es entwickelt sich folgender Dialog: „Oberkellner: Bitte sich Etwas auszusuchen, mein Herr. Der Deutsche [vor leerem Teller sitzend]: Es ist mir Alles Eins, was Sie mir von diesen Sachen bringen, aber bringen 'Sie nur endlich was. Oberkeller: Ja wissen Sie, es ist halt ein Umstand bei uns; haben thun wir Alles - aber fertig ist gar Nichts! - Der Deutsche 【auf das im Hintergrund sichtbare ,Gasthaus zum Zigeuner' deutend, wo einem Gast in ungarisicher Tracht auf einem Teller eine auffällig große Wurst serviert wird]: Hätt' ich das gewußt, so wär' ich in das andere Wirthshaus daneben gregangen; dort haben's zwar nur eine Extrawurst - aber die haben's halt das ganze Jahr." ${ }^{34}$

Der Hinweis auf die „Extrawurst“ zielt auf die verfassungsrechtliche Sonderstellung Ungarns infolge des Ausgleichs mit Kaiser und Regierung, der dem Land eine dauerhafte Rückkehr zum Konstitutionalismuss gebracht hat. Die Karikatur widerspiegelt die Verfassungssituation in der Habsburgermonarchie zur Jahresmitte 1867, als für die Länder der ungarischen Krone mit Beginn dieses Jahres die Wiederherstellung der 1848 geschaffenen Verfassung erfolgte; Ungarns - sich schon seit Oktoberdiplom und Reichsverfassung 1860/61 anbahnende - verfassungsrechtliche Sonderstellung im habsburgischen Gesamtstaat wurde dadurch fast zu staatlicher Unabhängigkeit: Unter einem gemeinsa-

\footnotetext{
${ }^{3}$ Kikeriki; Nr. 28 vom 11 .7. 1867, 4 [http://anno.onb.ac.at/cgi-content/anno?aid= kik\&datum $=18670711 \&$ seite $=4 \&$ zoom $=33$ ] (abgerufen 30. 7. 2013).
} 
men Monarchen war es mit den anderen - sogenannten cisleithanischen - Ländern der Habsburger aber weiterhin verbunden durch gemeinsame Angelegenheiten, nämlich Äußeres und Heerwesen. Während der ungarische Teil dieser Zwei-Staaten-Monarchie seit Jahresbeginn auf verfassungsstaatlichen Grundlagen ruhte, verharrte Cisleithanien, oder wie dieses nun amtlich hieß, „die im Reichsrat vertretenen Königreiche und Länder", auf dem Boden der neuständischen Verfassung von 1860/61: ohne umfassende Bindung des Monarchen an eine Verfassung, ohne Verantwortlichkeit der Minister dem Parlament gegenüber, ohne strikte Gewaltentrennung zwischen Justiz und Verwaltung, ohne umfassenden Grundrechtsschutz des Einzelnen, ohne Einrichtungen zur Kontrolle des Staates. $\mathrm{Zu}$ allem Überdruss kam noch hinzu, dass der Reichsrat nach der seit 1861 andauernden Weigerung der Landtage der ungarischen Länder, Delegierte in den Reichsrat nach Wien zu entsenden, im September 1865 sistiert worden war, um es Kaiser und Regierung zu ermöglichen, Verhandlungen mit den Vertretern der ungarischen Länder über deren künftige verfassungsrechtliche Stellung zu führen. ${ }^{5}$

\section{Anpassung der}

\section{cisleithanischen Verfassung an die Wirkungen des Ausgleichs}

\subsection{Vorlagen der Regierung an den Reichsrat}

Dem Ergebnis der Ausgleichsverhandlungen musste - infolge veränderter Stellung des Reichsrats und der Notwendigkeit der Bildung von Parlamentsdelegationen für die Handhabung der gemeinsamen Angelegenheiten - aber

\footnotetext{
${ }^{5}$ Zur Verfassungssituation in der Habsburgermonarchie zwischen 1861 und Ausgleich BRAUnEDER, Verfassungsentwicklung 169ff; AÖRG, Rechts- und Verfassungsgeschichte 216-221.
}

auch in der Verfassungsordnung Cisleithaniens Rechnung getragen werden. Der seit September 1865 sistierte Reichsrat wurde nach Neuwahl der Delegierten durch die cisleithanischen Landtage im Mai 1867 wieder einberufen, als engerer Reichsrat, also ohne Abgeordnete aus Transleithanien. Mitte Juni brachte die Regierung im Abgeordnetenhaus mehrere Vorlagen zur Anpassung der Verfassung Cisleithaniens an die Wirkungen des Ausgleichs ein: und zwar ein Gesetz über die Ministerverantwortlichkeit, ein Gesetz über -die durch den Ausgleich bedingten - Änderungen des Grundgesetzes über die Reichsvertretung, inklusive einer Novellierung des kaiserlichen Notverordnungsrechts, sowie ein Gesetz über die allen Ländern der österreichischen Monarchie gemeinsamen Angelegenheiten und die Art ihrer Behandlung, das später sogenannte Delegationengesetz. ${ }^{6}$

\subsection{Initiativen im Abgeordnetenhaus}

\subsubsection{Einsetzung eines Verfassungs- ausschusses - Bestellung von Subkomitees}

Im Abgeordnetenhaus,7 wo die Verfassungsvorlagen der Regierung am 19. Juni 1867 eingebracht worden waren, ${ }^{8}$ wurde zur Klärung der Frage der Behandlung der Regierungsvorlagen ein neunköpfiger Unterausschuss eingesetzt. ${ }^{9}$ Nur wenige Tage später lag am 21. Juni bereits sein Bericht vor: ${ }^{10}$ Er schlug die Zuweisung aller vier Vorlagen an einen besonderen Ausschuss zur Vorberatung vor; dieser sollte aus 36 Mit-

\footnotetext{
${ }^{6}$ AÖRG, Rechts- und Verfassungsgeschichte 222; BRAUNEDER, Verfassungsentwicklung 1ff.

${ }^{7}$ BRAUnEDER, Grundrechte 275ff; STOURZH, Dezemberverfassung $1 \mathrm{ff}$.

8 StenProtAH, 9. Sitzung, 17. 6. 1867, 183.

${ }^{9}$ Ebd. 10. Sitzung, 19. 6. 1867, 214f; von den Mitgliedern gehörten - außer Cölestin Leonardi - alle dann auch dem Verfassungsausschuss an, daher auch Sturm, der spätere Referent für die Ausarbeitung der Grundrechte: ebd. 216.

${ }^{10}$ Ebd. 11. Sitzung, 21. 6. 1867, $225 \mathrm{ff}$.
} 
gliedern bestehen und so zusammengesetzt sein, so dass alle im Reichsrat vertretenen Länder durch einen Abgeordneten repräsentiert waren, das Sitzrecht sollte aber allen Mitgliedern des Hauses eingeräumt sein.

Der Verfassungsausschuss wurde am folgenden Tag gewählt; ${ }^{11}$ zur Behandlung der Regierungsvorlagen betraute er spezielle Subkomitees, zunächst am 21. Juni 1867 eines für die Vorberatung des Gesetzes über die Verantwortlichkeit der Minister und kurz darauf am 27. Juni 1867 ein weiteres für die Änderungen des Grundgesetzes über die Reichsvertretung und das Ausgleichsgesetz, das sog. Delegationengesetz. Der zur "Revision der Verfassung überhaupt“ bestimmte Unterausschuss bestand zunächst aus sieben Mitgliedern; später kamen - „zur Verstärkung des Subkomitees“ - zwei weitere Mitglieder hinzu. ${ }^{12}$

\subsection{2. "Grundsätze " für die "Revision der Verfassung" - Eduard Sturm: Referent für die Grundrechte}

Nachdem das Gesetz über die Verantwortlichkeit der Minister am 23. Juli 1867 beide Häuser des Reichsrats, also auch die Erste Kammer, das Herrenhaus, erfolgreich passiert hatte, sah man im Verfassungsausschuss des Abgeordnetenhauses die Chance, über die Vorlagen der Regierung hinaus mit Ungarn verfassungsrechtlich gleichzuziehen. Nach Ansicht des Verfassungsausschusses waren die konkreten Verfassungsarbeiten im Subkomitee für die Verfassungsrevi-

\footnotetext{
11 StenProtAH, 12. Sitzung, 22. 6. 1867, 231.

${ }^{12}$ HAIDER, Protokolle 167f.; später, am 24.7. 1867, wurden „zur Verstärkung des Subcommités" zwei weitere Mitglieder gewählt: HAIDER, Protokolle 173f. Mitglieder waren die Abgeordneten Rudolf Brestel (NÖ), Sebastian Froschauer (Vorarlberg) als Obmann, Moriz Kaiserfeld (Steiermark), Franz Klier (Böhmen), Ignaz Kuranda (NÖ), Eduard Sturm (Mähren), Florian Ziemialkowski (Galizien) sowie ab 24.7. Rafael Kremer-Auenrode (OÖ) und Josef Waser (Steiermark).
}

sion vorzubereiten, wofür nur wenige Tage später „Grundzüge, nach denen das Subkomitee [...] bei der Revision der Verfassung vorzugehen hätte“, beschlossen wurden. ${ }^{13}$ Dieser Vorschlag zielte darauf $a b$, über die Regierungsvorlagen hinausgehend, weitere, als Staatsgrundgesetze zu bezeichnende, Verfassungsgesetze vorzubereiten, wodurch die von der Regierung intendierte „Revision“ des Februarpatents von 1861 zu einer völlig neuen Verfassung führen sollte, und zwar zu einer solchen im konstitutionellen Sinn. Es konnte so - vor allem dem Monarchen gegenüber - der Anschein erweckt werden, dass es bloß zu einer Ergänzung des seit 1861 bestehenden Verfassungsstatus kommen werde, und zwar dadurch, dass sich an das Grundgesetz über die Reichsvertretung aus 1861 weitere Verfassungsgesetze als Staatsgrundgesetze angliederten. Nach Empfehlung der "Grundzüge" sollten sich Verfassungsausschuss und Subkomitee bei der inhaltlichen Ausgestaltung der neuen Verfassungsgesetze als Vorbild „an die [...] Verfassung [...] 1849 [...] halten“" (Grundzüge, Punkt II). Damit kam jene „Entwicklung ins Rollen“ (Brauneder), die zu Jahresende 1867 auch für den staatlichen Verband der ReichsratsLänder die Rückkehr zum Konstitutionalismus bringen wird. ${ }^{14}$

Für die Ausarbeitung eines GrundrechteEntwurfs zeichnete Eduard Sturm verantwort-

\footnotetext{
${ }^{13}$ HAIDER, Protokolle 193ff; STOURZH, Dezemberverfassung 5; publiziert in der Wiener Zeitung, Nr. 175 vom 24. 7. 1867, Extra-Blatt zur Abend-Beilage. - Ein lithographiertes Exemplar des Originals in HHStA, Nachlass Plener, Kart. 44, Konvolut mit dem „Nachlass Sturm“ (Zeitungsausschnitte, Korrespondenzen, Widmungen, insbesondere in einem zerschlissenen Umschlag mit der Aufschrift „Reichsrath 1867. Verfassungsausschuss" Aufzeichnungen und Notizen zu den Arbeiten von Sturm im „Revisions Comité des Verfassungsausschusses".

${ }^{14}$ STOURZH, Dezemberverfassung 4; BRAUNEDER, Verfassungsentwicklung 174.
} 
lich,15 jüngstes Mitglied des Unterausschusses und Abgeordneter der Stadtgemeindekurie des mährischen Landtags. Sturm, ein gebürtiger Brünner, war 1861 als ungarischer Landesadvokat von Pest nach Brünn versetzt worden, nachdem er infolge der verfassungsrechtlichen Wende von 1860/61 als österreichischer Justizfunktionär in Ungarn - wie es damals hieß - „disponibel" geworden war und das Land verlassen musste. 1865 kandidierte er im Wahlbezirk Iglau [Jihlava] für ein zum Landtag erledigtes Mandat und wurde mit überwältigender Mehrheit, nämlich mit 210 von 217 Stimmen, darunter auch acht Frauenstimmen, wie im Landtagsprotokoll vermerkt ${ }^{16}$ wurde, gewählt. 1867 konnte er bei der auf die Beendigung der Sistierung des Reichsrates erfolgten Neuwahl des Landtags seinen Wahlerfolg wiederholen, er wurde nun mit 410 von 430 Stimmen gewählt; sein Gegenkandidat, der damals amtierende Bürgermeister von Iglau, hatte bloß 19 Stimmen erhalten.17 Ende Februar 1867 erfolgte die Wahl zum Delegierten der Stadtgemeindekurie des mährischen Landtags zum (engeren) Reichsrat in Wien. Anlässlich der Debatte über eine Adresse des Landtags an den Kaiser aus Anlass der Einberufung des Reichsrates äußerte sich Sturm zustimmend zum Ausgleich sowie zu dem auf bestimmte Angelegenheiten „,begränzten Dualismus“; er begrüßte auch die in Ungarn erfolgte Rückkehr zum Konstitutionalismus sowie die sich für Cisleithanien dadurch eröffnende Möglichkeit zur Entwicklung seiner Verfassung. ${ }^{18}$

Sturm konnte sich seit 1865 im Landtag als Abgeordneter der Deutsch-Liberalen profilieren, ${ }^{19}$

\footnotetext{
${ }^{15}$ STOURZH, Dezemberverfassung 10; LuFT, Sturm 13; Plener, Dr. Eduard Sturm; WurzBaCH, Biographisches Lexikon 40, 224-227.

${ }^{16}$ Mährisches LTB1. 1865, 2. Sitzung (24. 11. 1865), 27.

${ }^{17}$ Ebd. 1867, 2. Sitzung, 15 (19.2. 1867), 8. Sitzung (28. 2. 1867), 167.

${ }^{18}$ Mährisches LTBl. 1867, 7. Sitzung (27. 2. 1867), 148151, insbesondere 151.

${ }^{19}$ MALǏ̌, Biografický 676ff.
}

stand dort aber - noch - im Schatten von Karl Giskra, damals auch Bürgermeister von Brünn. In Wien zählte Giskra, ein sogenannter „1848er“, neben Adolf Pratobevera zu den Wortführern der Deutsch-Liberalen im Reichsrat. ${ }^{20}$ Unmittelbar vor Eröffnung des Reichsrates war Giskra vom Kaiser zum Präsidenten des Hauses ernannt worden; ${ }^{21}$ er war es dann auch, der den zur "Revision der Verfassung“ berufenen Ausschuss als "Verfassungsausschuss" bezeichne$t^{22}$, womit er eine Analogie zum Konstituierenden österreichischen Reichstag von 1848/49 bewusst machen wollte. ${ }^{23}$

Die in der Frage der "Revision der Verfassung“ federführende politische Fraktion des Abgeordnetenhauses, die liberale "Verfassungspartei", verfügte im Verfassungsausschuss über eine satte Mehrheit.24 Ihre Wortführer kamen aus

${ }^{20}$ GotTSMAnN, Reichstag und Reichsrat 632, 637f.

${ }^{21}$ Mitteilung von Ministerpräsident Beust in der Eröffnungssitzung am 20.5.1867: StenProtAH, 1. Sitzung (20. 5. 1867), 1. - Unter den beiden Vizepräsidenten befand sich mit Florian Ziemialkowski noch ein zweiter „1848er", er war später auch Mitglied des Verfassungsausschusses.

22 Ebd. 229.

${ }^{23} \mathrm{Zu}$ dieser Analogie BRAUNEDER, Verfassungsentwicklung 172f., 175f. Die Reichsratsmehrheit und Regierung sahen das nicht so: STOURZH, Dezemberverfassung 6f. Vier ehemalige Abgeordnete des Reichstags von 1848/49 (Gschnitzer, Potocki, Ziemialkowski, Brestel) gehörten 1867 dem Reichsrat an, die beiden zuletzt Genannten auch dem VerfassungsAusschuss.

${ }^{24}$ Ungeachtet der Abstinenz der (14) Tschechen vom Reichsrat verfügte die „linke“ liberal-zentralistischfortschrittliche Verfassungspartei - sie bestand, von wenigen Ausnahmen abgesehen, nahezu ausschließlich aus Deutsch-Liberalen, - mit 118 Stimmen nicht über die für Verfassungsänderungen erforderliche Zweidrittelmehrheit, ihr stand eine „rechte“ klerikalföderalistische-konservative Opposition mit 68 Stimmen gegenüber: RUMPLER, Parlament 702. Lediglich die Novellierung des Staatsgrundgesetzes über die Reichsvertretung erforderte eine qualifizierte Verfassungsmehrheit, für die Erzeugung der neuen Staatsgrundgesetze war die einfache Mehrheit ausreichend: $\mathrm{Zu}$ der ausführlichen Debatte über diese Frage im 
dem Kreis der deutsch-liberalen Abgeordneten; sie trafen regelmäßig auch außerhalb des Parlaments im privaten Kreis zusammen. Hinweise darauf finden sich etwa in den Memoiren des böhmischen Industriellen Carl Wolfrum, ${ }^{25}$ "graue Eminenz" der Liberalen im Reichsrat, ${ }^{26}$ dem Sturm als "der geistreichste" unter seinen Parteifreunden galt, ${ }^{27}$ oder in den Tagebüchern von Adolf Pratobevera, ${ }^{28}$ bei dem Sturm in der entscheidenden Phase der Verfassungsrevision neben anderen deutsch-liberalen Abgeordneten - zu "Spiel und Souper“ eingeladen war. Bei der Konstituierung des Verfassungsausschusses ${ }^{29}$ wurde Pratobevera ${ }^{30}$ als einer der erfahrensten deutsch-liberalen Abgeordneten zum Obmann gewählt; Sturm, der mit deutlicher Mehrheit (mit 135 von 153 Stimmen) Mitglied des Verfassungsausschusses und dann auch Mitglied des Subkomitees (mit 21 von 26 Stimmen) geworden worden war, fungierte als (erster) Schriftführer. ${ }^{31}$

Zuge der 2. Lesung des StGG-RiG im Plenum des Abgeordnetenhauses: PAAR, Gesetzgebung 80ff.

${ }^{25}$ WOLFRUM, Erinnerungen I, 424 (mehrere Treffen Ende Mai bei „Domayer“, Anwesenheit Pratobeveras und Giskras).

${ }^{26}$ HeIss, SteKL, URBANITSCH, Zusammenfassung 498, Anm. 46.

${ }^{27}$ WOLFRUM, Erinnerungen I, 302.

${ }^{28}$ HHStA, Familienarchiv Pratobevera, Kart. 13, Tagebücher "August 1867-15. Oktober 1868", hier Eintrag zum 11.10. („Spiel bei mir. Berger, Schindler, Herbst, Rechbauer, Waser, Winterstein, Sturm“), zum 11. 11. und 6. 12. („, bei mir zu Spiel und Souper [...] Die Abgeordneten: Berger [...], Brestl [...], Dienstl [...], Eichhof $[\ldots]$, Giskra [...], Herbst [...], Kremer [...], Sturm [...], Waser [...], Winterstein [...], Wickenhof $\left.[\ldots]^{\prime \prime}\right)$.

${ }^{29}$ HAIDER, Protokolle 166ff. passim.

30 BÖCK, Pratobevera 246f; HÖBELT, Pratobevera 676f.

${ }^{31}$ HAIDER, Protokolle 225ff., 228. - Eine Liste sämtlicher Mitglieder des Verfassungsausschusses und ihrer Funktionen ebd. 267f. im Anhang.

\section{Ablauf der Verfassungs- arbeiten in Verfassungs- ausschuss und Subkomitee}

Die Verfassungsarbeiten im Verfassungsausschuss und im Subkomitee zerfielen in drei Phasen. In der ersten Phase befassten sich die beiden Gremien bis 19. Juli 1867 mit Fragen, welche die „Vorberatung über die Behandlung der die Verfassung betreffenden Regierungsvorlagen“ betrafen. Hierzu wurde vom Subkomitee ein von Sturm gemeinsam mit Eduard Herbst ${ }^{32}$ ausgearbeiteter „Entwurf für die Anträge des Ausschusses" vorbereitet. Unmittelbar nach Annahme der Regierungsvorlagen über die Änderung des Notverordnungsrechts und die Ministerverantwortlichkeit in beiden Häusern des Reichsrates wurde im Verfassungsausschuss das weitere Vorgehen in der Frage der Verfassungsrevision auf Grundlage der inzwischen durch das Subkomitee bis Ende Juni vorbereiteten "Grundzüge, nach welchen bei Revision der Verfassung vorzugehen sei“, ${ }^{\prime \prime}$ erörtert: Vorgesehen war die Festlegung auf eine Revision der bestehenden Reichsverfassung durch Änderung des Grundgesetzes über die Reichsvertretung und dessen Ergänzung durch "Spezialgesetze“ als neue "Grundgesetze" (Punkt I); als solche waren vorgesehen ein "Gesetz über die allgemeinen Rechte der Staatsbürger", ein "Gesetz

\footnotetext{
${ }^{32}$ Herbst hatte sich unter den Deutsch-Liberalen aus Böhmen "eine beherrschende Stellung erkämpft": HÖBELT, Kornblume 20; er war "Gründer und führende Persönlichkeit“ der Deutsch-Liberalen im Böhmischen Landtag: URBAN, Landtag, 1999. Zu Herbst ferner WURZBACH, Biographisches Lexikon 8, 360f.; ANONYMUS, Herbst 280f.; KANN, Herbst 588f.; HÖBELT, Parteien 799ff. passim.

${ }_{33}$ HAIDER, Protokolle 105, 191ff., 193ff.; der Text des Konzepts ebd. 192f. Abdruck in NFP, Nr. 1039 vom 24. 7. 1867, Morgenblatt; ein lithographiertes Exemplar befindet sich auch im Nachlass Plener im HHStA, Kart. 44. Dazu auch STOURZH, Dezemberverfassung 5. Zum Ablauf dieser Phase der Entstehung der Verfassung 1867 auch STURM, Sprachenfrage 1.
} 
über die Rechte der Krone“ (später „über die Regierungs- und Vollzugsgewalt"), ein "Gesetz über die richterliche Gewalt" und ein "Gesetz über einen Reichsgerichtshof" (später „über die Einsetzung eines Reichsgerichts“). Bei diesen Gesetzen „würde es sich empfehlen, [...] die betreffenden Bestimmungen der Verfassung [...] $1849^{\prime \prime}$ als Vorbild zu nehmen, hieß es in Punkt II;34 im Grundgesetz über die Reichsvertretung sollte gemäß Punkt I - außer den von der Regierung vorgeschlagenen Änderungen bei Festhalten am Delegationsprinzip auch eine Revision des Wahlrechts (Änderung des Mandatsverteilungsschlüssels auf die Länder sowie Erhöhung der Zahl der Abgeordneten von bisher 203 auf 300), ferner eine Erweiterung der sogenannten "Länderautonomie" durch taxative Fixierung der Reichsratszuständigkeiten und Zuweisung von Kompetenzen in Gemeindeangelegenheiten und im Bereich des Schulwesens Berücksichtigung finden.

Die Aufnahme von konkreten Verfassungsarbeiten wurde bis zur Vorlage entsprechender Entwürfe durch das Subkomitee unterbrochen; die zur Verfassungsreform erforderlichen Vorarbeiten wurden auf einzelne Mitglieder des Subkomitees verteilt. ${ }^{35}$ Nachdem die erforderli-

\footnotetext{
${ }^{34}$ Es wurde auch empfohlen, den Verfassungsentwurf von Kremsier (Rechbauer) bzw. die April-Verfassung 1848 (Gschnitzer) zu berücksichtigen: HAIDER, Protokolle 191. STURM, Sprachenfrage 2 meinte dazu, es sei dies eine "von ihm selbst angeregte Richtschnur" gewesen.

${ }^{35}$ Dazu HaIDER, Protokolle 195ff. passim sowie $261 \mathrm{f}$. (Verzeichnis der Mitglieder des Verfassungsausschusses). Lediglich zwei Subkomiteemitglieder, Franz Klier und Ignaz Kuranda, sowie der Obmann des Subkomitees, Sebastian Froschauer, wurden nicht als Referenten bzw. Berichterstatter eingesetzt. Am stärksten in Anspruch genommen war Josef Waser als Berichterstatter für die Novellierung des Notverordnungsrechts, für das StGG-RiG und das StGG-ARVG sowie über das Kundmachungsgesetz zur Verfassung 1867. Rudolf Brestel war Berichterstatter für das Delegationengesetz und - gemeinsam mit Florian Ziemialkowski - auch mit der Ausarbeitung der Novellie-
}

chen Entwürfe ausgearbeitet und im Subkomitee einer ersten Durchsicht und Überarbeitung unterzogen worden waren, kamen Mitte September auch die Arbeiten im Verfassungsausschuss wieder in Gang. ${ }^{36}$

Und damit setzte die zweite Phase der Entstehung der Verfassung 1867 ein: Von 17. bis 21. September befasste sich der Verfassungsausschuss in mehreren Sitzungen zunächst mit den Entwürfen zum Staatsgrundgesetz über die richterliche Gewalt, über die Regierungs- und Vollzugsgewalt sowie über die Einsetzung eines Reichsgerichts ${ }^{37}$, am 21. September schließlich auch mit dem Staatsgrundgesetz über die allgemeinen Rechte der Staatsbürger. Sturm erstattete zunächst einen allgemeinen Bericht, dann erfolgte die Verlesung und die Spezialdebatte über die $\S \S 1$ bis 17 seines im Subkomitee bereits umgearbeiteten Entwurfs. Nach reger Debatte über $\S 17$, betreffend die Rechte der Angehörigen der einzelnen Nationalitäten, wurde der Entwurf zur Neuformulierung dieser Bestimmungen an das Subkomitee zurückverwiesen. Die Beratungen konnten dann nicht wie geplant schon am 23., sondern erst am 25. September fortgesetzt, dann aber auch zügig bis $\S 19$, der Schlussbestimmung des Grundrechtskatalogs, abgeschlossen werden. ${ }^{38}$ Von 1 . bis 6. Oktober schloss sich die Vorberatung der Novelle zum Grundgesetz über die Reichsvertretung an, womit die zweite Phase der Ausarbeitung der Ver-

rung des StGG-RV betraut, wofür Moriz Kaiserfeld als Berichterstatter fungierte; Raphael KremerAuenrode war Berichterstatter für das StGG-RG, und Eduard Sturm schließlich fungierte als solcher für das StGG-ARStB.

${ }^{36}$ Es wurden drei neue Schriftführer bestellt (HAIDER, Protokolle 196), sodass sich Sturm noch mehr als in der ersten Phase auf die konkreten Verfassungsarbeiten konzentrieren konnte.

${ }^{37}$ HAIDER, Protokolle 198ff., 201ff., 206ff., $209 f$.

${ }^{38}$ Ebd. 215. 
fassung 1867 im Verfassungsausschuss abgeschlossen war. ${ }^{39}$

Nach Abschluss der Vorberatungen im Verfassungsausschuss wurden die Entwürfe des Verfassungsausschusses am 5. Oktober $1867 \mathrm{im}$ Plenum des Abgeordnetenhauses eingebracht; mit den Grundrechten befasste sich das Haus am 8. und 9. Oktober. ${ }^{40}$ Im Herrenhaus wurden die Beschlüsse des Abgeordnetenhauses ab 22. Oktober eingebracht; 41 dort wurden sie einer aus den Mitgliedern von zwei schon bestehenden Kommissionen zusammengesetzten Kommission, der „vereinigten juridisch-politischen Commission",42 zur Vorberatung zugewiesen. ${ }^{43}$ Im Plenum befasste sich das Herrenhaus mit den Grundrechten am 28. November. ${ }^{44}$ Nach Rücküberweisung der von ihm beanstandeten und abgeänderten Bestimmungen des Staatsgrundgesetzes über die allgemeinen Rechte der Staatsbürger an das Abgeordnetenhaus am 4. Dezember 186745 befasste sich zunächst dessen Verfassungsausschuss noch am selben Tag 46 sowie anschließend am 7. Dezember wieder das Plenum mit den Grundrechten. ${ }^{47}$ Nach Rücküberweisung des Grundrechte-Entwurfs - wegen einer noch bestehenden Differenz - an das Herrenhaus war nach dessen Einverständnis am 12. Dezember $1867^{48}$ ein übereinstimmender Beschluss der beiden Häuser des Reichsrats zum

\footnotetext{
${ }^{39}$ Ebd. 219ff., 224ff., 228ff., 231f., $236 \mathrm{ff}$.

40 StenProtAH, 32. und 33. Sitzung, 8. bzw. 9. 10. 1867, 778ff. bzw. $799 f f$.

${ }^{41}$ Ebd. 13. Sitzung, 22. 10. 1867, 173.

${ }^{42}$ Ebd. 14. Sitzung, 30. 10. 1867, 212f. (Konstituierung).

${ }^{43}$ In der "Vereinigten juridisch-politischen Commission" als Mitglieder unter anderem auch die "1848er" Schmerling und Miklosich): Liste der Mitglieder in StenProtHH, Index, 198f. bzw. 199f. (Judicielle bzw. Politische Commission).

${ }^{44}$ Ebd. 17. Sitzung, 28. 11. 1867, 267-288.

${ }^{45}$ StenProtAH, 58. Sitzung, 4. 12. 1867, 1571.

${ }^{46}$ HAIDER, Protokolle 260ff.

${ }^{47}$ StenProtAH, 59. Sitzung, 7. 12. 1867, 1598ff.

${ }^{48}$ StenProtHH, 21. Sitzung, 12. 12. 1867, 37; Mitteilung im AH: StenProtAH, 64. Sitzung, 13. 12. 1867, 1810.
}

Staatsgrundgesetz über die allgemeinen Rechte der Staatsbürger zustande gekommen.

Nach übereinstimmenden Beschlüssen über das Kundmachungsgeset ${ }^{49} \mathrm{zu}$ den Regierungsvorlagen (Novelle zum Grundgesetz über die Reichsvertretung und Delegationengesetz) sowie $\mathrm{zu}$ den vier Staatsgrundgesetzen am 21. Dezember 1867 erhielten alle sieben Gesetzesbeschlüsse des Reichsrates am folgenden Tag die Sanktion des Monarchen; mit der zugleich erfolgten Kundmachung 50 standen die neue Verfassung und mit ihr auch die neuen Grundrechte in Kraft. ${ }^{51}$

\section{Entstehung des Grundrechtekatalogs}

\subsection{Der "erste" Entwurf Eduard Sturms}

Eduard Sturm ${ }^{52}$ begann die Ausarbeitung des Entwurfs für das Staatsgrundgesetz über die allgemeinen Rechte der Staatsbürger wohl unmittelbar nach der Ende Juni 1867 erfolgten Vertagung der Verfassungsarbeiten in Verfassungsausschuss und Subkomitee.

Aufgrund seiner Verpflichtungen als Anwalt in Brünn nahm er die Ausarbeitung des Textes wohl in seiner Heimatstadt vor; jedenfalls erreichte ihn dort am 4. September 1867 ein Telegramm mit der Aufforderung, dass er sich zu den am 17. September beginnenden Verhandlungen des Verfassungsausschusssubkomitees wieder in Wien einfinden solle. ${ }^{53}$

\footnotetext{
${ }^{49}$ RGBl. 1867/147.

${ }^{50}$ RGBl. 1867/141-146.

${ }^{51}$ RGBl. 1867/142.

52 BRAUNEDER, Grundrechte 277f.; DERS., Verfassungsentwicklung 174ff., besonders 181f.; STOURZH, Dezemberverfassung $10 f$.

${ }^{53} \mathrm{Im}$ "Nachlass" Sturms, fol. 70: siehe sogleich Anm. 51. STURM, Sprachenfrage 1, weist auch auf diese „Privataufzeichnungen" hin.
} 
Die von ihm hinterlassenen Materialien mit Aufzeichnungen ${ }^{54}$ über die Beratungen im „Revisions Comité" erlauben einen Blick auf seine Arbeitsweise:

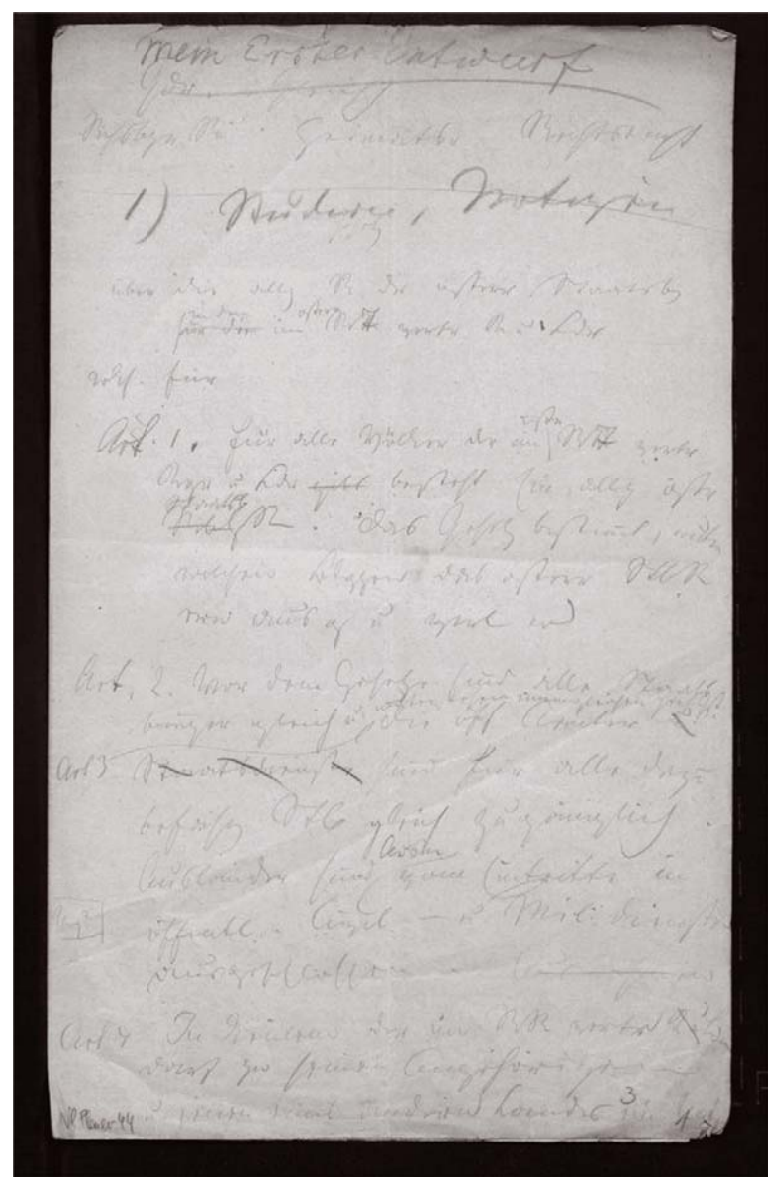

Er konzipierte zunächst mit Bleistift in flüchtiger Handschrift, zum Teil unter Verwendung von eigenwilligen Abkürzungen ${ }^{55}$, einen Katalog von

\footnotetext{
${ }^{54}$ Sein „Nachlass“ bildet einen Bestandteil des umfangreichen, mehrere Dutzend Kartons umfassenden NL Plener im ÖStA, HHStA; Ernst Plener, ein Deutsch-Böhme, war einer der Führungsfiguren des deutsch-liberalen Lagers seit Mitte der 1870er Jahren, u.a. 1893/94 auch Finanzminister (GOLDINGER, Plener 122f.; RUMPLER, Plener 529f.); sein Vater war Ignaz Plener, 1860/65 Finanzminister, 1867 Mitglied des Verfassungsausschusses, dann 1867/68 Handelsminister und schließlich Vertreter der Altliberalen im Herrenhaus; in Kart. 44 finden sich auch mehrere, meist ungeordnete Konvolute zu Sturms Arbeiten im Parlament, u.a. eines mit der Aufschrift „Reichsrath 1867. Verfassungsausschuss“, das 92 durchfoliierte Blätter umfasst, die Blätter 54-65 fehlen allerdings.

55 Ebd. fol. 3-6.
}

Grundrechten; als „Mein Erster Entwurf“ bezeichnet; er enthält in 25 Artikeln folgende Grundrechte: 56

Art. 1: Einheitliches Staatsbürgerrecht für „die Völker" der Reichsratsländer;

Art. 2: Gleichheit aller Staatsbürger vor dem Gesetz; gleicher persönlicher Gerichtsstand; Art. 3: Gleicher Zugang der Staatsbürger zu öffentlichen Ämtern; Ausnahmen für Ausländer;

Art. 4: Einheit des Privat-, Straf- und Verfahrensrechts in allen Ländern; gleiche Steuerpflicht der Staatsbürger;

Art. 5: Freizügigkeit (von Person und Vermögen); Beschränkung der Auswanderungsfreiheit durch Wehrpflicht; Abfahrtsgelder bei Reziprozität zulässig;

Art. 6: Eigentumsfreiheit; Enteignung nur gegen Entschädigung;

Art. 7: Aufenthalts- und Wohnsitzfreiheit, Freiheit des Liegenschaftserwerbs und der Berufsausübung; Freiheit der Teilung des Grundeigentums; Zulässigkeit der Beschränkung von Erwerb und Verfügung über Liegenschaften durch Kirche oder geistliche Stiftungen;

Art. 8: Aufhebung von Grunduntertänigkeit und Hörigkeit; Grundentlastung;

Art. 9: Freiheit der Person; Zuerkennung von Schadenersatz bei rechtsiwidriger Verhaftung;

Art. 10: Grundsatz staatlicher Gerichtsbarkeit; Grundsatz des mündlich-öffentlichen Gerichtsverfahrens; Anklageprozess in Strafsachen; Einrichtung von Schwurgerichten; Unterstellung von Heeresangehörigen unter die staatliche Zivilgerichtsbarkeit;

Art. 11: Abschaffung der Todesstrafe; Aufhebung der Strafe des bürgerlichen Todes, der Vermögenskonfiskation, des Prangers, der Brandmarkung, Kettenstrafe und körperlichen Züchtigung;

Art. 12 und 13: Schutz des Hausrechts sowie des

${ }^{56}$ Ebd. fol. $2 \mathrm{ff}$. 
Briefgeheimnisses;

Art. 14 und 15: Petitionsrecht sowie Vereinsund Versammlungsfreiheit;

Art. 16: Glaubens- und Gewissensfreiheit einschließlich des Rechts der häuslichen Religionsausübung; Unabhängigkeit der bürgerlichen und politischen Rechte vom Glaubensbekenntnis;

Art. 17: Öffentliche Religionsübung und Zuerkennung innerer Autonomie für staatlich anerkannte Bekenntnisse; keine Vorrechte für bestimmte Bekenntnisse;

Art. 18: Kein Zwang zu kirchlichen Handlungen oder Feiern;

Art. 19: Gesetzliche Regelung des Verhältnisses des Staates zu den Religionsgesellschaften;

Art. 20: Grundsatz der obligatorischen Ziviltrauung; Aufhebung des Ehehindernisses der Religionsverschiedenheit;

Art. 21: Freiheit der Wissenschaft und ihrer Lehre; Freiheit der Gründung von Schulen und Erziehungsanstalten;

Art. 22: Allgemeine Volksbildung als Staatsaufgabe; Gleichbehandlung der Volksgruppen in multinationalen Ländern im öffentlichen Unterricht; Grundsatz der einheitlichen Unterrichtssprache; Organisierung des Religionsunterrichts durch Kirchen und Religionsgesellschaften; staatliche Leitung und Aufsicht über das Unterrichts- und Erziehungswesen;

Art. 23: Rede-, Presse-, Meinungsfreiheit; Aufhebung der Zensur;

Art. 24: Gleichbehandlung der Nationalitäten in Bezug auf Nationalität und Sprache;

Art. 25: Zulässigkeit der Suspension von bestimmten Grundrechten.

In den von Sturm nachgelassenen Materialien finden sich auch die von ihm auf dem ersten Blatt seines ersten Entwurfs in der Überschrift erwähnten "Studien und Notizen",57 nämlich

\footnotetext{
${ }^{57}$ Ebd. fol. 3r.
}

über die Grundrechte der Verfassung 1849, 58 des Kremsierer Reichstags ${ }^{59}$ und der Frankfurter Nationalversammlung aus 1848/49 sowie von mehreren deutschen Einzelstaaten (aus der Zeit des Vormärz bzw. aus den Jahren 1848 bis 1850) und den Niederlanden aus $1848 .{ }^{60}$ Es finden sich außerdem Notizen über die Grundrechtsdiskussion im Kremsierer Reichstag, ${ }^{61}$ ferner ein lithographiertes Exemplar der vom Verfassungsausschuss am 23. Juli 1867 beschlossenen „Grundzüge, nach welchen bei Revision der Verfassung vorzugehen sei", 62 samt Notizen über die Debatten im Verfassungsausschuss.

Auf der Grundlage dieser Materialien verfasste Sturm eine Reinschrift seines ersten Entwurfs, betitelt als "Gesetz über die allgemeinen Rechte der Staatsbürger für die im Reichsrathe vertretenen Königreiche und Länder",63 und zwar in Form einer zweispaltigen Tabelle: der Gesetzestext selbst ist in der rechten Spalte enthalten und mit dunkler Tinte geschrieben, in der linken Spalte brachte Sturm mit Bleistift zu den einzelnen Artikeln und Absätzen fortlaufend Anmerkungen an - gezählt nach ", $x^{\prime \prime}$, , $x x^{\prime \prime}$ und "xxx", worin er auf Parallelstellen in anderen Verfassungstexten hinwies. Regelmäßig finden sich Angaben zu den entsprechenden Paragrafen im Kremsierer Verfassungsentwurf, ${ }^{64}$ in den

\footnotetext{
${ }^{58}$ Ebd. fol. 7f.: „1849 G[run]dR[echte] über die d[urc]h die const[itutionelle] Staatsform gewährleisteten Rechte“ (Grundrechtspatent RGBl. 1849/11).

${ }^{59}$ Ebd. fol. 9f.: „Oest: Grundrechte (= Entwurf der Grundrechte des österreichischen Volkes, in Wiener Zeitung, Nr. vom 23. 12. 1848/Extrablatt).

${ }^{60}$ Ebd. fol. 11f.: „Frankfurt [...] Niederlande 1848 [...] Kurhessen 1831 [...] Hannover $1840 \mathrm{u}$ [nd 18]48 [...] Oldenburg 1849 [...] Bremen 1849 [...] Preussen 1850“.

${ }^{61}$ Ebd. fol. 13: „Debatte 1848-49-Grundrechte“ von „3/1 49.“ bis „26/1“, also von 3. bis 26. Jänner 1849, im Kremsierer Verfassungsausschuss.

62 Ebd. fol. 15: „Grundzüge“.

${ }^{63}$ Ebd. fol. 17-21.

${ }^{64}$ Z.B.: „1848 I.“ als Hinweis auf $§ 1$ der Kremsierer Grundrechte bei Art. 1, Abs. 2 von Sturms Entwurf.
} 
Frankfurter Grundrechten ${ }^{65}$ und der Verfassung 1849,66 zum Teil mit Angaben über inhaltliche Details; 67 Verweise auf Parallelstellen in anderen Verfassungstexten sind selten, ein paar Mal wird die preußische Verfassung von 1850 angeführt, bloß einmal wird auf die ungarische Verfassung von 1848 hingewiesen. ${ }^{68}$ Unberücksichtigt blieben - ungeachtet einer Empfehlung im Verfassungsausschuss - die Grundrechte der Verfassung 1848.69 Unklar ist die Bedeutung der Abkürzung „Rel. Ed.“ unter Sturms Anmerkungen zu einzelnen Bestimmungen seines Entwurfs. ${ }^{70}$

${ }^{65}$ Z.B.: „Fkf. § 7“ als Hinweis auf $\S 7$ der Grundrechte der Deutschen von 1848 bei Art. 2 von Sturms Entwurf.

${ }^{66}$ Z.B.: „1849 §. 23 V.“ als Hinweis auf § 23 der Reichsverfassung 1849 bei Art. 1, Abs. 1 von Sturms Entwurf, bzw. "1849 § 10“ ohne das ergänzende Kürzel „V.“ als Hinweis auf $\S 10$ des Grundrechtepatents für Cisleithanien aus 1849 bei Art. 5, Abs. 1 von Sturms Entwurf.

${ }^{67}$ Z.B.: „Fkf. $\theta$ (jeder Deutsche)" als Hinweis auf das Fehlen einer Bestimmung über die Einheitlichkeit der Staatsbürgerschaft im Konzept der Grundrechte der Frankfurter Nationalversammlung; oder „1848 Amend[ment]. Mayer" als Hinweis auf die auf Antrag von Cölestin Mayer in den Kremsierer Verfassungsentwurf (§ 138) aufgenommene Schadenersatzpflicht des Staates für durch Beamte zugefügte Grundrechtsverletzungen als Hinweis zu Art. 9, Abs. 3 von Sturms Entwurf.

${ }^{68}$ Z.B. zur preußischen Verfassung: "Pr. $\S 4$ " bei Art. 3, Abs. 1 von Sturms Entwurf, oder „preuss[ische]. V[er]f[a]ss[un]g. 37" bei Art. 11 von Sturms Entwurf.; z.B. zur ungarischen Verfassung: „ung[arisches]. G[esetz-]Bl. XXIII. §2" als Hinweis auf den Gesetzartikel XXIII der ungarischen Verfassung 1848 bei Art. 11 von Sturms Entwurf.

${ }^{69}$ Bleistiftnotiz von Sturm über diesen Vorschlag von Gschnitzer auf dem in seinem "Nachlass" befindlichen Exemplar der "Grundzüge“ zu Punkt II. In den Protokollen des Verfassungsausschusses findet sich darüber kein Hinweis: HAIDER, Protokolle 191, Anm. b.

70 Z.B. zu Art. 3, 6, 7, 15, 17 und 20. „Rel.Ed“ könnte für „Religionsedikte“ stehen, ein Terminus, der auch als Synonym für die sogenannten „konfessionellen“ Gesetze verwendet wurde, welche in Verbindung mit dem Abbau des Konkordats von 1855 im Abgeordne-

\section{2. Überarbeitung des Entwurfs Sturm im Subkomitee des Verfassungsausschusses}

Nach Wiederzusammentritt des Verfassungsausschusses wurde Sturms „erster" Entwurf Anfang September 1867 im Subkomitee einer Überarbeitung unterzogen:71 Es kam insbesondere zur Streichung der Bestimmungen über besondere Aspekte der Gleichheit der Staatsbürger (persönlicher Gerichtsstand; gleiches Zivil-, Straf- und Verfahrensrecht; gleiche Steuerpflicht), der Bestimmungen über die Justiz (Gerichtsorganisation; Grundsätze des Zivilund Strafverfahrens; Zuständigkeit ordentlicher Gerichte für Militärpersonen in Zivilsachen) und der Bestimmungen über das Strafrecht (Aufhe-

tenhaus seit 1867 in Ausarbeitung begriffen waren, und an welchen Sturm federführend beteiligt war: Darauf weist auch STURM, Sprachenfrage 2, hin. - Ein solcher Hinweis findet sich auch in der eingangs illustrierten Karikatur.

${ }^{71}$ BRAUNEDER, Grundrechte 278f. Sturm hat diese Veränderungen im Exemplar der Reinschrift seines Entwurfs mit Bleistift ersichtlich gemacht, durch Streichungen bzw. inhaltliche Hinweise, welche er zum Teil unmittelbar bei den entsprechenden Bestimmungen (bei Art. 4/gleicher Beitrag $\mathrm{zu}$ den "Staatslasten" durch den Hinweis auf "Gemeindegenossen" bzw. bei Art. 10/staatliche Gerichtsbarkeit durch den Hinweis auf "Niemand darf seinem ord[entlichen] Richter entzogen werden" oder bei Art. 23/Verbot der Zeitungszensur anstatt des Terminus "Zeitungsstempel“: "Concessionssyst[em].“) angebracht hat bzw. in einer stichwortartigen Aufzählung in dem in seinem "Nachlass" befindlichen Konvolut "Reichsrath 1867. Verfassungsausschuss", hier fol. 21v (Aufzählung von Materien, welche in „Spez[ial-].Gesetze“ gehören: „Unab[ängigkeit]. der Richter", mit Verweis auf den Satz "Niemand d[ar]f s[eine]m ord[entlichen]: Richter entzogen werden", der sich auf $\S 1$ des Gesetzes zum Schutz der persönlichen Freiheit bezieht, wo anstelle von "ordentlichen" freilich "gesetzlichen“ steht; ferner: "Schadenersatz des Staates" im Rahmen der "Administr[ativ].Justiz[-]Conflicte“, „Lehen. 1862“, „Heimathrecht 1862 u[nd] 1863“" [bezugnehmend auf die Gesetze über die Lehensallodifikation sowie das Gemeinde- und Heimatrechtswesen: RGBl. 103/1862, bzw. 18/1862 und 105/1863). 
bung der Todesstrafe und einiger anderer Strafarten; Zuständigkeit in Preß-Delikten) sowie der Bestimmungen betreffend die Beziehungen zwischen dem Staat und den von ihm anerkannten Kirchen (Aufhebung von Privilegien einzelner Konfessionen; gesetzliche Bestimmung der Verhältnisse zwischen Staat und Kirchen; Einführung der obligatorischen Zivilehe). Die betreffenden Gegenstände sollten zum Teil in anderen Staatsgrundgesetzen (über Gerichtsbarkeit bzw. über die Regierungs- und Vollzugsgewalt) eine Regelung erfahren, zum Teil in besonderen Gesetzen neben den Staatsgrundgesetzen. Überdies wurde ein neues Grundrecht geschaffen: Allen Staatsbürgern sollte in jenen Gemeinden, in denen sie einer Steuerpflicht unterlagen, das Wahlrecht zur Gemeindevertretung und zum Landtag zugestanden werden. Außerdem kam es bei einzelnen Bestimmungen zu stilistischen Änderungen und Kürzungen im Wortlaut sowie zu Modifikationen ihrer systematischen Anordnung. Der Großteil von Sturms Entwurf blieb aber unverändert. Er wurde infolge der Streichungen im Subkomitee im Umfang von 25 auf 19 Artikel reduziert. ${ }^{72}$

\subsection{Der Entwurf des Subkomitees und seine Veränderungen im Verfassungs- ausschuss sowie im Plenum der beiden Häuser des Reichsrates}

Die gegen Ende September 1867 anschließenden Verhandlungen im Verfassungsausschuss ${ }^{73}$ brachten - von stilistischen Modifikationen und

\footnotetext{
${ }^{72} \mathrm{Im}$ Konvolut „Reichsrath 1867. Verfassungsausschuss", fol. 22v seines "Nachlasses") findet sich in Bleistift die Anweisung Sturms: „nur das mit Tinte Geschriebene gilt u[nd]. erbitte ich mir dieses Manuscript wegen der Bleistiftnotizen zurück". Ein gedrucktes Exemplar des Subkomitee-Entwurfs befindet sich im "Nachlass" Sturm in NL Plener im Konvolut „Reichsrath 1867. Verfassungsausschuss“, fol. 23-25. ${ }^{73}$ BRAUNEDER, Grundrechte 279; HAIDER, Protokolle 118ff und 209ff; $121 \mathrm{ff}$ und 214ff.
}

systematischen Umstellungen ${ }^{74}$ abgesehen kaum wesentliche Änderungen - ausgenommen einerseits die Streichung des Verbots der unbeschränkten Teilbarkeit von Grundeigentum (Art. 6, Abs. 2 des Subkomitee-Entwurfs) sowie der Bestimmung über die Einheitlichkeit der Unterrichtssprache in multinationalen Ländern (Art. 17, Abs. 3 des Subkomitee- Entwurfs); andererseits wurde auch ein neues Grundrecht kreiert, der Grundsatz der Freiheit der Berufswahl (Art. 18 des VerfassungsausschussEntwurfs). ${ }^{75}$

Die Verhandlungen im Plenum der beiden Häuser des Reichsrats (von Anfang Oktober bis Mitte Dezember 1867) in Verbindung mit den anderen Entwürfen zu den Verfassungsgesetzen, welche die neue Verfassung bildeten, führten zu keinen wesentlichen inhaltlichen Änderungen des Verfassungsausschuss-Entwurfs. ${ }^{76}$ Als Referent des Entwurfs im Verfassungsausschuss und Plenum des Abgeordnetenhauses konnte Eduard Sturm weitergehende inhaltliche Änderungen und formale Umstellungen in der Gliederung seines Konzepts der Grundrechte zum Großteil abwehren. ${ }^{77}$ Die von ihm zu Beginn der zweiten Lesung als Berichterstatter im Abgeordnetenhaus getroffenen allgemein-staatsrechtlichen Ausführungen über Funktion der Grundrechte geben tiefe Einblicke in das zeitgenössische Verständnis der Liberalen über das Wesen

\footnotetext{
${ }^{74}$ HAIDER, Protokolle 210-218 passim.

${ }^{75}$ HAIDER, Protokolle 214, $217 \mathrm{f}$.

${ }^{76}$ BRAUNEDER, Grundrechte 276f, 277ff; HAIDER, Protokolle 122f, 124ff, 257ff, 261f; StenProtAH, 32. Sitzung, 9.10.1867, 780-795; ebd. 33. Sitzung, 8. 10. 1867, 799-818; ebd. 58. Sitzung, 4. 12. 1867, 1571; ebd. 59. Sitzung, 7. 12. 1867, 1598ff, 1626f; StenProtHH, 17. Sitzung, 28.11.1867, 267-288; ebd. 21. Sitzung, 12.12.1867, 373; ebd. 22. Sitzung, 13. 12. 1867, 388ff. Die Sanktion erfolgte am 21. 12. 1867: RGBl. 141-147.

77 HAIDER, Protokolle 122f; BRAUNEDER, Grundrechte $277 \mathrm{ff}$.
} 
der Grundrechte in einem freiheitlichen Verfassungsstaat. ${ }^{78}$

Die im Anhang (7.) folgende Synopse veranschaulicht die im Verfassungsausschuss sowie im Plenum der beiden Häuser des Reichsrates an der durch das Subcomité beschlossenen Fassung des Entwurfs Sturm: Die in der linken Spalte durchgestrichenen Passagen weisen auf Textstreichungen hin; kursiv gesetzte Passagen betreffen inhaltliche Änderungen. Die rechte Spalte enthält die jeweilige Artikelnummer der im Beschluss des Reichsrates verbliebenen Bestimmungen; die Artikelzählung liegt auch dem nach der am 21. Dezember 1867 erfolgten kaiserlichen Sanktion kundgemachten Text des Staatsgrundgesetzes über die allgemeinen Rechte der Staatsbürger zugrunde.

\section{Ergebnis}

Der Verfassungsausschuss kann - durch die Initiative zum Ausbau der Verfassung im konstitutionellen Sinn - als „Träger und Motor der Verfassungsreform" von 1867 angesehen werden. Er ist dadurch „zum eigentlichen Schöpfer" der neuen Verfassung geworden. Ihr Herzstück - die Grundrechte - wurden sogar als das „ureigenste Werk“ des Verfassungsausschusses angesehen. Das ihnen zugrundeliegende Konzept war Sturms „erster" Entwurf, sodass auch er als „Schöpfer" des Grundrechte-Katalogs von 1867 angesehen werden könnte. ${ }^{79}$ Allerdings hatte er sich bei der Ausarbeitung seines Entwurfs sehr stark an früheren Konzepten orientiert, vor allem an den Grundrechten der österreichischen Verfassung von 1849. Am Schluss seiner "Studien und Notizen", die er der Ausarbeitung seines Entwurfes zu Grunde gelegt hatte, brachte Sturm die lapidare Bemerkung "Alles 1849“

\footnotetext{
${ }^{78}$ StenProtAH, 32. Sitzung, 8. 10. 1867, 778ff.

${ }^{79}$ STOURZH, Dezemberverfassung 4.
}

$a^{80}$ - sie gibt die materielle Essenz seines Entwurfs freilich sehr stark verkürzend wieder: Er hat - außer dem cisleithanischen GrundrechtePatent - sogar den Grundrechten des Kremsierer Reichstags Beachtung geschenkt ${ }^{81}$ und vereinzelt auch den Frankfurter Grundrechten von 1848/49 den Vorzug82 gegeben. Zwei Grundrechte waren überdies durch strafrechtliche Nebengesetze aus 1862 inhaltlich bereits festgelegt, nämlich der Schutz der persönlichen Freiheit sowie der Schutz des Hausrechts. ${ }^{83}$

Die Fassung, welche der Entwurf Sturms im Subkomitee erhalten hatte, wurde in den weiteren Beratungen weder im Verfassungsausschuss noch im Plenum der beiden Häuser des Reichsrats wesentlichen Änderungen in Bezug auf Inhalt und System unterzogen. Sturm kann daher durchaus als der "eigentliche Schöpfer" jedenfalls der Textgrundlage - des Grundrechtekatalogs von 1867 angesehen werden, ${ }^{84} \mathrm{im}$ Hinblick auf die Methode der Verwertung der von ihm benutzten Vorlagen aber wohl „doch eher nur im formellen Sinn als Kompilator" ${ }^{.85}$

\footnotetext{
${ }^{80}$ Bleistiftnotiz Sturms auf der Rückseite eines Blattes, das im Anschluss an die Reinschrift seines Entwurfs in dem mit "Reichsrath 1867. Verfassungsausschuss" bezeichneten Konvolut in seinem „Nachlass“, hier fol. $21^{\mathrm{v}}$.

${ }^{81}$ Dem Kremsierer Entwurf folgen Art. 19 (Gesetzliche Regelung des Verhältnisses von Staat und Kirche) und Art. 22 Abs. 2 (Einheitlichkeit der Unterrichtsprache) von Sturms Entwurf.

${ }^{82}$ Beschränkungen der "toten Hand“ (Art. 7, Abs. 2 in Sturms Entwurf); Aufhebung der Todesstrafe (Art. 11 in Sturms Entwurf), Verbot des Zwangs zu kirchlichen Handlungen oder Feiern (Art. 18 in Sturms Entwurf).

${ }^{83}$ Gesetz zum Schutz der persönlichen Freiheit (Art. 9 in Sturms Entwurf) bzw. Gesetz zum Schutz des Hausrechts (Art. 12 in Sturms Entwurf), jeweils vom 27. 10. 1862, RGBl. 35 und 36.

${ }^{84}$ STOURZH, Dezemberverfassung 10.

${ }^{85}$ BRAUNEDER, Grundrechte 278.
} 


\section{Anhang: Synopse}

Entwurf Subkomitee: Änderungen im Verfassungsausschuss

Reichsratsbeschluss

Art. 1 Einheitliches Staatsbürgerrech

Art. 2 Gleichheit aller Staatsbürger vor dem Gesetz

Art. 3 Gleiche Befähigung zu öffentlichen Ämtern;

Ausnahmen für Ausländer

Art. 4 (1) Freizügigkeit (von Person und Vermögen);

(2) Verknüpfung von Steuerleistung am Wohnsitz und Wahlrecht;

(3) Beschränkung der Auswanderungsfreiheit durch Wehrpflicht;

(4) Abfahrtsgelder bei Reziprozität zulässig

Art. 5 Eigentumsfreiheit; Enteignung nur gegen Entschädigung

Art. 6 (1) Freiheit von Aufenthalt / Wohnsitz, Verfügung über Liegenschaften und Ausübung jeden Erwerbszweigs;

(2) Grundsatz der Teilbarkeit von Grundeigentum;

(3) $=(2)$ Beschränkung des Liegenschaftserwerbs für die "tote Hand“

Art. 7 Aufhebung von Grunduntertänigkeit und Hörigkeit; Grundentlastung

Art. 8 Freiheit der Person; Schadenersatz bei rechtswidriger Verhaftung

Art. 9 Schutz des Hausrechts

Art. 10 Schutz des des Briefgeheimnisses

Art. 11 Petitionsrech

Art. 12 Vereins- und Versammlungsfreiheit

Art. $13=$ Art. 14

(1) Glaubens- und Gewissensfreiheit

(2) Unabhängigkeit der Rechtsstellung vom

Glaubensbekenntnis

(3) Kein Zwang zu kirchlichen Handlungen oder Feiern

Art. $14=$ Art. 15 Öffentliche Religionsübung und Anerkennung innerer

Autonomie für staatlich anerkannte Bekenntnisse

Art. $15=$ Art. 16 Recht der häuslichen Religionsausübung

Art. $16=$ Art. 17

(1) Freiheit der Wissenschaft und ihrer Lehre;

(2) Gründung von Schulen / Erziehungsanstalten nach Gesetz;

(3) Freiheit des häuslichen Unterrichts

(4) Organisierung des Religionsunterrichts durch Kirchen

(5) staatl. Leitung und Aufsicht über Unterricht / Erziehung

Art. $17=$ Art. 19

(1) Gleichberechtigung der Volksgruppen in Bezug auf Nationalität und Sprache;

(2) [1.]Gleichbehandlung der Volksgruppen im öffentlichen Unterricht inklusive

[2.]Verbot des Sprachenzwangs;

(3) Grundsatz der einheitlichen Unterrichtssprache

Art. $18=$ Art. 13 Rede-, Presse-, Meinungsfreiheit; Aufhebung der Zensur

Art. 18 Recht auffreie Berufswahl

Art. $19=$ Art. 20 Suspension bestimmter Grundrechte

$=$ Art. 7

$=$ Art. 8

$=$ Art. 9

$=$ Art. 10

$=$ Art. 11

$=$ Art. 12

$=$ Art. 14

$=$ Art. 15

$=$ Art. 16

$=$ Art. 17

$=$ Art. 19

$=(1)$

(2) Gleichberechtigung der Landessprachen;

$=(3)$

$=$ Art. 13

$=$ Art. 18

$=$ Art. 20 


\section{Korrespondenz:}

Dr. Christian Neschwara

Universität Wien

Institut für Rechts- und Verfassungsgeschichte

Schottenbastei 10-16, 1010 Wien

christian.neschwara@univie.ac.at

\section{Abkürzungen:}

$\begin{array}{ll}\text { AÖRG } & \text { Arbeitsgemeinschaft } \\ & \text { Österreichische Rechtsgeschichte } \\ \text { LTB1 } & \text { Landtagsblatt } \\ \text { NFP } & \text { Neue Freie Presse } \\ \text { NL } & \text { Nachlass } \\ \text { NÖ } & \text { Niederösterreich } \\ \text { OÖ } & \text { Oberösterreich } \\ \text { Rz. } & \text { Randziffer }\end{array}$

StenProtAH Stenographische Protokolle über die Sitzungen des Hauses der Abgeordneten des österreichischen Reichsrates (1861ff)

StenProtHH Stenographische Protokolle über die Sitzungen des Herrenhauses des österreichischen Reichsrates (1861ff)

\section{Literatur:}

ANONYMUS, Herbst, Eduard, in: ÖBL, Bd. 2 (Wien 1959) $280 \mathrm{f}$.

AÖRG (Hg.), Rechts- und Verfassungsgeschichte (Wien 22012).

Brigitte BÖcK, Pratobevera von Wiesborn, Adolf, in: ÖBL, Bd. 8 (Wien 1981) $246 f$.

Wilhelm BRAUNEDER, Gesetzgebungsgeschichte der österreichischen Grundrechte, in: Rudolf MACHACEK, Willibald P. PAHR, Gerhard STADLER (Hgg.), 70 Jahre Republik. Grund- und Menschenrechte in Österreich. Grundlagen, Entwicklung und internationale Verbindungen (Kehl am Rhein-Straßburg-Arlington1991) 189-364.

Wilhelm BRAUNEDER, Verfassungsentwicklung in Österreich 1848 bis 1918, in: RUMPLER, URBANITSCH, Habsburgermonarchie VII/1, 69-237.

Walter GOLDINGER, Plener, Ernst, in: ÖBL, Bd. 8 (Wien 1981) $122 \mathrm{f}$.

Andreas GotTsmann, Der Reichstag 1848/49 und der Reichsrat 1861 bis 1865, in: RUMPLER, URBANITISCH, Habsburgermonarchie VII/1, 569-565.

Barbara HAIDER (Hg.), Die Protokolle des Verfassungsausschusses des Reichsrates vom Jahre 1867
(=Fontes rerum austriacarum II/88, Wien-KölnWeimar 1997).

Hans HeIss, Hannes SteKL, Peter Urbanitsch, Zusammenfassung, in: Peter URBANITSCH, Hannes STEKL (Hgg.), Kleinstadtbürgertum in Cisleithanien 1862-1914 (= Bürgertum in der Habsburgermonarchie IX, Wien-Köln-Weimar 2000) 465-503.

Lothar HÖBELT, Kornblume und Kaiseradler. Die deutschfreiheitlichen Parteien Altösterreichs 18821918 (Wien 1993).

Lothar HÖBELT, Parteien und Fraktionen, in: RUMPLER, URBANITSCH, Habsburgermonarchie VII/1, 8951006.

Lothar HÖBELT, Pratobevera, Adolf, in: NDB, Bd. 20 (Berlin 2001) 676f.

Robert A. KANN, Herbst, Eduard, in: NDB, Bd. 8 (Berlin 1969) 588f.

Robert LuFT, Sturm, Eduard, in: ÖBL, Bd. 14 (Wien 2012) 13.

Jiři MALIŘ u.a. (Hg.), Biografický slvenik poslanců moravského zemského sněmu v letech 1861-1918 (Brünn 2012).

Martin PAAR, Die Gesetzgebung der österreichischen Monarchie im Spiegelbild der Normen und der staatsrechtlichen Literatur, (=Europäische Hochschulschriften, Reihe 2, Rechtswissenschaft 4792, Frankfurt am Main u.a. 2009).

Ernst Plener, Dr. Eduard Sturm, in: NFP, Nr. 16172 vom 29. 8. 1909, 2.

Helmut Rumpler, Peter Urbanitsch (Hgg.), Die Habsburgermonarchie 1848-1918, Bd. VII (Verfassung und Parlamentarismus), Teilbd. 1: Verfassungsrecht, Verfassungswirklichkeit, zentrale Repräsentativkörperschaften; Teilbd. 2: Die regionalen Repräsentativkörperschaften (Wien 2000).

Helmut RuMPLER, Parlament und Regierung Cisleithaniens 1867 bis 1914, in: DERS., URBANITSCH, Habsburgermonarchie VII/1, 667-894.

Helmut RuMPLER, Plener, Ernst, in: NDB, Bd. 20 (Berlin 2001) 529f.

Gerald STOURZH, Die österreichische Dezemberverfassung von 1867, in: Österreich in Geschichte und Literatur 12 (1968) 1-16.

Eduard STURM, Die Sprachenfrage in Österreich in: NFP, Nr. 14218 vom 25. 3. 1904, 1-3.

Otto URBAN, Der böhmische Landtag, in: RuMPLER, URBANITSCH, Habsburgermonarchie VII/2, 19912055.

Carl, Otto, Wilhelm und Ludwig WOLFRuM, Erinnerungen an Carl Wolfrum, 2 Bde. (Leizpig 1893).

Constant WURZBACH, Biographisches Lexikon des Kaiserthums Österreich, 60 Bde. (Wien 18561891). 